\title{
Environment generated quantum correlations in bipartite qubit-qutrit systems
}

\author{
Salman Khan* and Ishaq Ahmad \\ Department of Physics, COMSATS Institute of Information Technology, \\ Chak Shahzad, Islamabad, Pakistan.
}

(Dated: May 18, 2015)

\begin{abstract}
The dynamics of entanglement and quantum discord for qubit-qutrit systems are studied in the presence of phase damping and amplitude damping noises. Both one way and two couplings of the marginal systems with the environments are considered. Entanglement sudden death is unavoidable under any setup, however, the required time span depends on the way of coupling. On the other hand, the dynamics of quantum discord strongly depends both on the nature of environment and on the number of dimensions of the Hilbert space of the coupled marginal system. We show that freezing and invariance of quantum discord, as previously reported in the literature, are limited to some special cases. Most importantly, it is noted that under some particular coupling the existence of environment can guarantee the generation of nonclassical correlations.
\end{abstract}

PACS: 03.65.Ud; 03.65.Yz; 03.67.Mn;04.70.Dy

Keywords: Entanglement; Quantum discord; Decoherence;

*Electronic address: sksafi@comsats.edu.pk 


\section{INTRODUCTION}

Quantum mechanics is one of the theories that is more perplexing and yet very fascinating which provides a complete physical description to fundamental phenomenon taking place at the atomic levels. Indeterminism and nonlocality are the two fundamental and controversial concepts of quantum theory. In quantum theory the sources of nonlocality are AharonovBohm effect and entanglement. Entanglement has been recognized as the first candidate of non-classical correlations and is considered as a vital resource for quantum information science [1]. It has been widely investigated and a number of schemes have been presented for its detection, quantification and applications [2-4]

A generic quantum state $\rho$ is an object which is characterized not only by quantum but also by classical correlations. From quantum information point of view, distinguishing these two types of correlations is of utmost importance. One way to do this is to use entanglement versus separability formalism introduced by Werner [5]. Moreover, in a quantum state there exist correlations that are not captured by the measures of entanglement and are still useful in carrying out certain quantum information tasks. Quantum discord, with no classical analog, introduced by Ollivier and Zurek [6] and, independently, by Henderson and Vedral [7] for bipartite systems is a more general measure of quantum correlations which also captures those that do not come in the domain of quantum entanglement. It has been used as a resource for certain quantum computation models [8], encoding of information onto a quantum state [9] and quantum state merging [10, 11]. Being an important measure of quantum correlations, the domain of the measurement of quantum discord has been recently extended to continuous variable systems of Gaussian and non-Gaussian states [12 14]. The study of the behavior of quantum discord for a two mode squeezed state in noninertial frames shows that quantum discord asymptotes to zero in the limit of infinite acceleration

[15]. A detail of other studies related to quantum discord, alternative measures of quantum correlations and its behavior in different setups are discussed in Refs. [18-26].

The irreversible loss of fundamental quantum features such as quantum superposition by a quantum system when it interacts with an environment is one of the big issues in the practical implementation of different protocols based on these quantum features. The utility of entanglement in different quantum information tasks as a resource decreases when the system interacts for considerably long time with its environment. The effect of environ- 
ment on entanglement between the components of a composite system leads to a number of undesirable consequences, such as entanglement loss, entanglement sudden death and even the rebirth of entanglement [28 31]. The study of the dynamics of quantum entanglement and quantum discord in Markovian environment shows that, unlike entanglement, quantum discord is immune to sudden death [32]. A sudden transition between classical and quantum loss of correlations for a class of Bell diagonal states under local dephasing noise has also been reported [33]. This behavior predicts that there exists a finite time interval, in which only classical correlations decay and quantum discord is frozen despite the presence of a noisy environment. In Ref. [34] the authors have shown that depending on the initial state, the quantum discord, in the presence of non-Markovian purely dephasing environment, might get frozen forever at a positive value. The behavior of quantum discord and entanglement in the presence of local qutrit dephasing noise is studied in Ref. [35, 36]. It is shown that the entanglement for the system vanishes in a finite time interval but the quantum discord remain invariant throughout the time evolution.

In this paper, we study the behaviors of entanglement and quantum discord for a particular bipartite state, which consists of a qubit and a qutrit as marginal systems where each interacts with a local environment. The influences of two types of noisy environments, the dephasing and the amplitude damping noises are investigated. Both one way and two ways coupling of the marginal systems in the form of local and multilocal environments are considered. We find that the freezing and decay, as reported previously, in the presence of a noisy environment are not the only aspects of quantum discord. It is found that the behavior of quantum discord strongly depends on the dimensions of the Hilbert space of the marginal system. We show that maneuvering the type of coupling between the system and a particular environment can lead to the generation or destruction of quantum discord.

\section{MEASURES OF QUANTUM CORRELATIONS}

In this section we briefly review the quantifiers for entanglement and non classical correlations. Many entanglement measures for quantifying entanglement of bipartite states exist in the literature. However, we will use negativity which is a reliable measure of entanglement of bipartite states of any dimensions, provided that the state has a negative partial transpose. The partial transpose of a bipartite density matrix $\rho_{m \nu, n \mu}$ over the second qubit $B$ is given 
by $\rho_{m \mu, n \nu}^{T_{B}}=\rho_{m \nu, n \mu}$ and for the first qubit, it can similarly be defined. For a bipartite state $\rho^{A B}$, the negativity $\mathcal{N}\left(\rho^{A B}\right)$ is defined as twice the absolute sum of the negative eigenvalues of partial transpose of $\rho^{A B}$ with respect to the smaller dimensional system,

$$
\mathcal{N}\left(\rho^{A B}\right)=\sum_{i}\left|\lambda_{i}\right|-\lambda_{i}
$$

where $\lambda_{i}$ are the eigenvalues of the partial transposed density matrix.

The nonclassical correlation are quantified by discord. The discord $\mathcal{D}\left(\rho^{A B}\right)$ for a bipartite state $\rho^{A B}$ is defined as the difference between total correlations $I\left(\rho^{A B}\right)$ and the classical correlation $C\left(\rho^{A B}\right)$,

$$
\mathcal{D}\left(\rho^{A B}\right)=I\left(\rho^{A B}\right)-C\left(\rho^{A B}\right) .
$$

The quantum mutual information $I\left(\rho^{A B}\right)$ is a measure of total amount of classical and quantum correlations in a quantum state. Mathematically, it is given by

$$
I\left(\rho^{A B}\right)=S\left(\rho^{A}\right)+S\left(\rho^{B}\right)-S\left(\rho^{A B}\right),
$$

where $S(\rho)=-\operatorname{Tr}\left(\rho \log _{2} \rho\right)$ is the von Neumann entropy of the system in the state $\rho$ and $\rho^{A(B)}=\operatorname{Tr}_{B(A)}\left(\rho^{A B}\right)$ are the two marginal states of the composite system. The classical correlation by definition is the maximal information that one can obtain and is mathematically given by [6, 7]

$$
C_{B}\left(\rho^{A B}\right)=S\left(\rho^{B}\right)-\min _{\left\{\Pi_{k}^{A}\right\}} \sum_{k} p_{k} S\left(\rho_{k}^{B}\right),
$$

where $\rho_{k}^{B}=\operatorname{Tr}_{A}\left(\left(\Pi_{k}^{A} \otimes I^{B}\right) \rho^{A B}\left(\Pi_{k}^{A} \otimes I^{B}\right)\right) / p_{k}$ is the postmeasurement state of subsystem $B$ after obtaining the outcome $k$ on subsystem $A$ with probability $p_{k}=$ $\operatorname{Tr}\left(\left(\Pi_{k}^{A} \otimes I^{B}\right) \rho^{A B}\left(\Pi_{k}^{A} \otimes I^{B}\right)\right)$. The set $\left\{\Pi_{k}^{A}\right\}$ are projectors onto the space of marginal state $A$ and $I^{B}$ is the identity operator for the space of marginal state $B$ of the composite system. It is important to mention that for a general mixed state, the measure of classical correlations is not symmetric, that is, $C_{A}\left(\rho^{A B}\right) \neq C_{B}\left(\rho^{A B}\right)$, as a result, the quantum discord depends on which marginal state the projective measurement is carried on. However, it is known that $\mathcal{D}_{A}\left(\rho^{A B}\right), \mathcal{D}_{B}\left(\rho^{A B}\right) \geq 0$ and $\mathcal{D}_{A}\left(\rho^{A B}\right)=\mathcal{D}_{B}\left(\rho^{A B}\right)=0$ if and only if $\rho^{A B}$ is a classical-quantum state. We will investigate the quantity $C_{B}\left(\rho^{A B}\right)$ for qubit-qutrit states such that the projective measurement is made on the qubit marginal state of the composite system. The measurement operators $\Pi_{k}(k=1,2)$ in the qubit space can be expressed as 
follows

$$
\Pi_{k}=\frac{1}{2}\left(I \pm \sum_{j} n_{j} \sigma_{j}\right),
$$

where the \pm sign corresponds to $k=1,2$, respectively and $\sigma_{j}(j=1,2,3)$ are the three Pauli spin matrices. The vector $n$ defines a unit vector on Bloch sphere having components $n=(\sin \theta \cos \phi, \sin \theta \sin \phi, \cos \theta)^{T}$ with $\theta \in[0, \pi]$ and $\phi \in[0,2 \pi]$. The main obstacle in obtaining quantum discord for a general quantum state lies in the minimization procedure, which is taken over all possible von Neumann measurements, of the quantum conditional entropy of equation (4). The analytical expressions for classical correlation and quantum discord are only available for two-qubit Bell diagonal state and a seven-parameter family of two-qubit X states [16, 19] till now. For the simple qubit-qutrit states that we consider in our work, we will calculate the quantum discord via numerical minimization over the two independent real parameters $\theta$ and $\phi$.

\section{THE SYSTEM IN NOISY ENVIRONMENT}

We consider a composite system of a qubit $A$ and a qutrit $B$ such that the two marginal systems are locally or multilocally coupled to their environments. The local and multilocal couplings describe the situations when either the qubit or the qutrit or both the qubit and the qutrit are independently influenced by their own environments.

The interaction of a system with an environment is studied in terms of various quantum channels (noise) such as phase damping noise and amplitude damping noise. When the

density matrix of a system is influenced by a dephasing noise, the diagonal elements of the density matrix remain unaffected while the off-diagonal elements decay. The dynamics of quantum correlations of the system we have chosen for the present studies have been investigated in Ref. [? ] under the condition that only the qutrit is coupled locally to dephasing environment. In the first part of our study, we reconsider the effect of dephasing noise on the same system, however, in multilocal coupling and show that such coupling of the system with dephasing noise can result in generation of quantum discord and also can leave it noninvariant. The second part of our study explores the influence of amplitude damping noise on the dynamics of quantum correlations. It is shown that for a finite time both local and multilocal coupling with amplitude damping noise generate quantum discord, however, 
completely destroys it in the asymptotic limit. The easy way of studying the dynamics of an open quantum system is to use Kraus operator formalism. The Kraus operators for a single qubit and single qutrit dephasing noise are, respectively, given as

$$
\begin{aligned}
& E_{A 0}^{D}=\operatorname{diag}\left(1, \sqrt{1-\gamma_{A}(t)}\right), \quad E_{A 1}^{D}=\operatorname{diag}\left(0, \sqrt{\gamma_{A}(t)}\right), \\
& E_{B 0}^{D}=\operatorname{diag}\left(1, \gamma_{B}(t), \gamma_{B}(t)\right), \quad E_{B 1}^{D}=\operatorname{diag}\left(0, \sqrt{1-\gamma_{B}^{2}(t)}, 0\right), \\
& E_{B 2}^{D}=\operatorname{diag}\left(0,0, \sqrt{1-\gamma_{B}^{2}(t)}\right),
\end{aligned}
$$

where the time dependent parameters are given by $\gamma_{A}(t)=1-e^{-t \Gamma_{A}}$ and $\gamma_{B}(t)=1-e^{-t \Gamma_{B}}$. The $\Gamma_{i}(i=A, B)$ represent the decay rates of the two marginal systems. The Kruas operators for both single qubit and single qutrit amplitude damping noise are not all diagonal and are given by

$$
\begin{aligned}
& E_{A 0}^{A}=\left(\begin{array}{cc}
1 & 0 \\
0 & \sqrt{1-\beta_{A}(t)}
\end{array}\right), \quad E_{A 1}^{A}=\left(\begin{array}{cc}
0 & \sqrt{\beta_{A}(t)} \\
0 & 0
\end{array}\right), \\
E_{B 0}^{A}= & \left(\begin{array}{ccc}
1 & 0 & 0 \\
0 & \sqrt{1-\beta_{B}(t)} & 0 \\
0 & 0 & \sqrt{1-\beta_{B}(t)}
\end{array}\right), \quad E_{B 1}^{A}=\left(\begin{array}{ccc}
0 & \sqrt{\beta_{B}(t)} & 0 \\
0 & 0 & 0 \\
0 & 0 & 0
\end{array}\right), \\
E_{B 2}^{A}= & \left(\begin{array}{ccc}
0 & 0 & \sqrt{\beta_{B}(t)} \\
0 & 0 & 0 \\
0 & 0 & 0
\end{array}\right),
\end{aligned}
$$

where the time dependent parameters $\beta_{i}(t)$ are defined similar to the $\gamma_{i}$ 's along with decaying parameters as above. It is easy to see that both $\gamma_{i}, \beta_{i} \epsilon[0,1]$, where the lower limit corresponds to no coupling and the upper limit corresponds to complete coupling of the system and environment. The Kraus operators for both qubit and qutrit satisfy the completeness relation $\sum_{i} E_{i}^{\dagger} E_{i}=I$. The evolution of the initial density matrix of the system when it is influenced by the multilocal noise is given in the Kraus operators formalism as follows

$$
\rho(t)=\sum_{j=0}^{3} \sum_{k=0}^{2}\left(E_{B j} E_{A k}\right) \rho(0)\left(E_{A k}^{\dagger} E_{B j}^{\dagger}\right)
$$


where $E_{A k}=E_{A m} \otimes I_{3}, E_{B j}=I_{2} \otimes E_{B n}$ are the Kraus operators of the local coupling of the qubit and the qutrit individually. The system is said to be coupled multilocally when the marginal states are influenced simultaneously by their local environments. The subscripts $m=0,1$, and $n=0,1,2$ stand, respectively, for a single qubit and a single qutrit Kraus operators of dephasing or amplitude damping noise. The identity matrices $I_{2}$ and $I_{3}$ act, respectively, on the qubit and qutrit parts of the composite system. we consider the initial density matrix $\rho(0)$ of the composite system given by the following one parameter family of matrices

$$
\begin{aligned}
\rho(0) & =\frac{p}{2}(|00\rangle\langle 00|+| 01\rangle\langle 01|+| 12\rangle\langle 12|+| 11\rangle\langle 11|+| 01\rangle\langle 11| \\
& +|11\rangle\langle 01|+| 00\rangle\langle 12|+| 12\rangle\langle 00|)+\frac{1-2 p}{2}(|02\rangle\langle 02| \\
& +|02\rangle\langle 10|+| 10\rangle\langle 02|+| 10\rangle\langle 10|),
\end{aligned}
$$

where $p \in[0,0.5]$ and $\rho(0)$ is separable only when $p=1 / 3$.

We first analyze the case when only the qubit is locally coupled to the phase damping noise. Under this condition, the nonzero matrix elements of $\rho(t)$ are given by

$$
\begin{aligned}
& \rho_{11}=\rho_{22}=\rho_{55}=\rho_{66}=\frac{p}{2} \\
& \rho_{33}=\rho_{44}=\frac{1-2 p}{2} \\
& \rho_{16}=\rho_{25}=\rho_{52}=\rho_{61}=\frac{p e^{-t \Gamma_{A} / 4}}{2}, \\
& \rho_{34}=\rho_{43}=\frac{(1-2 p) e^{-t \Gamma_{A} / 4}}{2} .
\end{aligned}
$$

It can be seen from the above matrix elements that only the offdiagonal elements decay with time. To observe the behavior of entanglement, we take partial transpose of $\rho(t)$ over the qubit and using Eq. (1), the negativity becomes

$$
\mathcal{N}(\rho)=\frac{1}{2} e^{-\Gamma_{A} / 4}\left(\left(1-e^{\Gamma_{A} / 4}\right)(1-p)+\left|\left(2+e^{\Gamma_{A} / 4}\right) p-1\right|+\left|p-e^{\Gamma_{A} / 4}(1-2 p)\right|\right) .
$$

The quantum mutual information is given by

$$
\begin{aligned}
I(\rho) & =\frac{1}{\log 4}\left[2 e^{-t \Gamma_{A} / 4}\left(\tanh ^{-1}\left(e^{-t \Gamma_{A} / 4}\right)-p \tanh ^{-1}\left(p e^{-t \Gamma_{A} / 4}\right)\right)\right. \\
& +4 \tanh ^{-1}\left(\frac{p}{3 p-2}\right)-p(\log 4+4 \log (1-2 p) \\
& \left.\left.-2 \log \left(p+p^{2}\right)\right)+\log \left(4+\frac{4\left(p^{2}-1\right)}{e^{-t \Gamma_{A} / 2}-p^{2}}\right)\right] .
\end{aligned}
$$


The analytical relation for quantum conditional entropy is very large, which results in very lengthy and complicated relation for classical correlation $C_{B}(\rho)$. This in turn gives a complicated relation for quantum discord which is impossible to be optimized analytically. Therefore, we will resort to numerical optimization for studying the behavior of quantum discord for all cases of coupling between the system and environment that we consider in this paper.

\section{NUMERICAL ANALYSIS}

For the purpose of numerical optimization, we first study the case when only the qubit is locally coupled to dephasing environment. Figure 1( $a$ ) shows the behaviors of negativity and quantum discord against the dimensionless parameter $t \Gamma_{A}$ for two values of the state parameter $p=0.15,0.23$. It can be seen that for both choices of $p$, the decoherence disentangles the system in finite time, however, the time domain in which the complete loss of entanglement occurs is different for each value of $p$. Comparatively, it survives longer (blue (dotted) curve) for $p=0.15$ than for $p=0.23$ (green (dash-dotted) curve). Although quantitatively different, this behavior of entanglement is qualitatively similar to what is reported in the case of local coupling of the qutrit with dephasing environment [36]. On the other hand, the dynamics of quantum discord strongly depend on which marginal system is locally coupled to dephasing environment. Unlike the results of Ref. [36], which shows invariance quantum discord for $p=0.23$, we find that for both values of $p$ the decay in quantum discord (black (solid) and red (dashed) curves) happens, however, at different instants of time. The time span for which discord remains freezed increases with the value of $p$. The remarkable aspect is that there exists a critical value of time at which quantum discord becomes independent of the state parameter $p$. The dynamics of quantum correlations of the system under multilocal coupling with dephasing environment are shown in figure $1(b)$. The blue (dotted $p=0.15$ ) and the green (dashed-dotted $p=0.23$ ) curves represent the behavior of entanglement. We see that for small values of $p$ the entanglement sudden death occurs very quickly and there is no entanglement at all for large values of $p$. Regardless of the value of $p$, the discord (black (solid) and red (dashed) curves) is always larger than the entanglement and exists in the system beyond the instant at which entanglement loss has taken place. The solid curve $(p=0.15)$ shows that in the presence of multilocal environment the discord monotonically decreases without being freezed for any interval. 


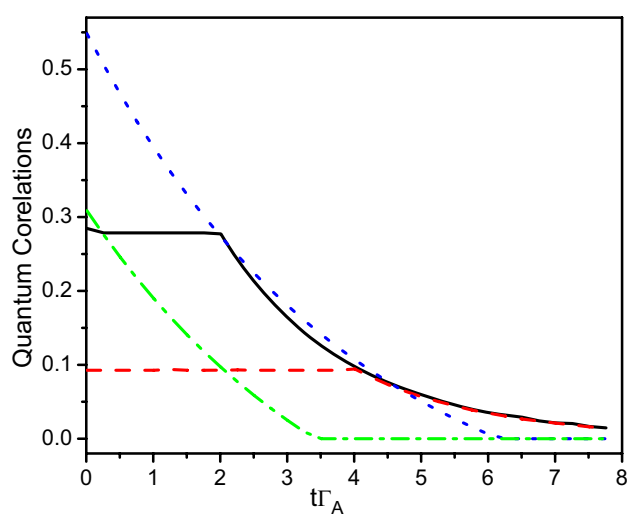

(a)

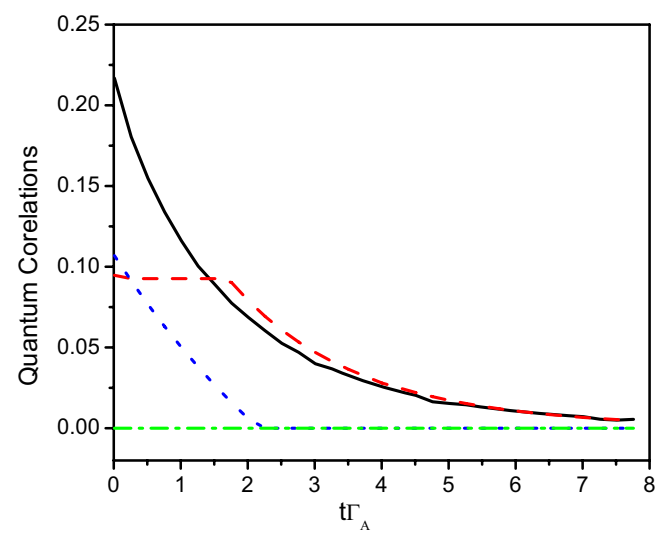

(b)

FIG. 1: (Color Online) Both the negativity (blue dotted, green dash-dotted) and the quantum discord (black solid, red dashed) are plotted, respectively, for two values of $p=\left(\begin{array}{lll}0.15, & 0.23)\end{array}\right.$ against the dimensionless parameter $t \Gamma_{A}$. (a) Shows the dynamics of correlations when only the qubit is locally coupled to dephasing noise. (b) Shows the situation for $t \Gamma_{B}=2$ when both the marginal systems are coupled locally to dephasing noise.

Figure 2 shows the dynamics of quantum correlations in multilocal coupling for the same two values of $p$ against the dimensionless parameter $t \Gamma_{B}$, which defines the qutrit's local environment. Unlike the effect of qubit's environment on the dynamics of discord (solid and dashed curves), we see that neither there is instantaneous decay nor the decay is as fast as in figure 1b. Most importantly, for $p=0.23$ (dashed curve) the discord is static initially, then abruptly increases, and remains almost static for the rest of the time. This behavior shows the robustness of discord against the local coupling of the qutrit marginal system with its environment. In fact, this is not only the case with discord, a comparison with figure $1 \mathrm{~b}$ shows that entanglement (dashed and dash-dotted curves) is also comparatively robust in this setup. We also confirm that our results reduce to the results of Ref. [? ] for $\Gamma_{A}=0$.

Next, we numerically analyze the dynamics of entanglement and quantum discord in the presence of amplitude damping noise. In figure $3 a$, we show the dynamics of quantum correlations when only either the qubit or the qutrit is locally coupled to its own environment. 


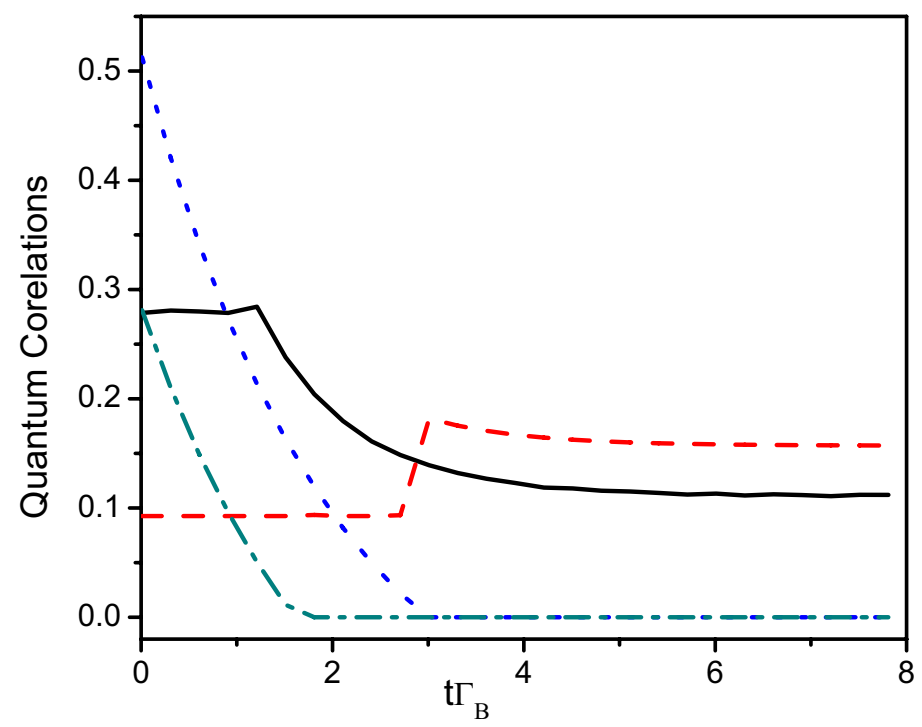

FIG. 2: (Color Online) Both the negativity (blue dotted, green dash-dotted) and the quantum discord (black solid, red dashed) are plotted, respectively, for two values of $p=(0.15,0.23)$ against the dimensionless parameter $t \Gamma_{B}$ for $t \Gamma_{A}=2$ when both the marginal systems are coupled locally to dephasing noise.

The green (dotted) and the black (solid) curves represent the behaviors of entanglement and quantum discord when only the qubit is locally coupled to its environment. The blue (dash-dot) and the red (dash) curves show the dynamics of entanglement and quantum discord, respectively, when only the qutrit is locally influenced by its environment. One can note that regardless of which marginal system is coupled to its local environment, the loss of entanglement is qualitatively identical. The same is true for quantum discord in the limit of large values of the plotting parameters. However, in the range of small values of $t \Gamma_{i}$, the behavior of quantum discord strongly depends on which marginal system is coupled to its local environment. The qubit's environment initially generates nonclassical correlations in the system that grow smoothly and then monotonically decays reaching 


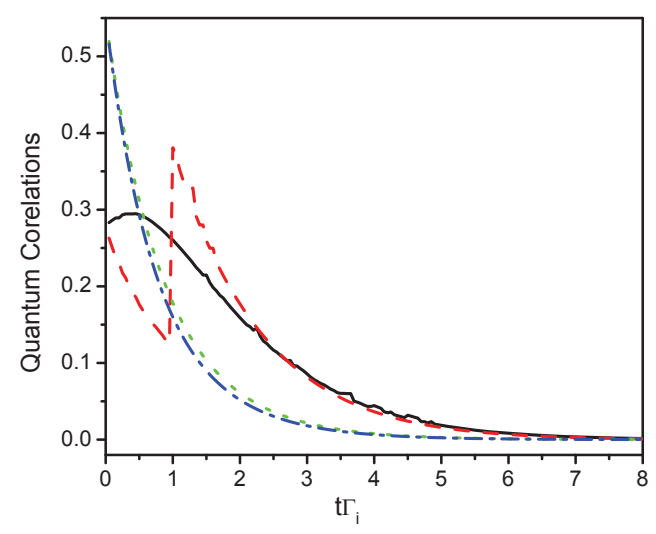

(a)

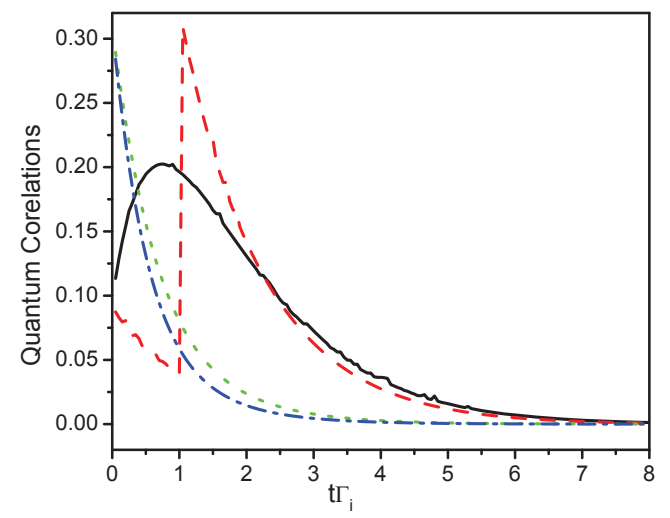

(b)

FIG. 3: (Color Online) Both the negativity and the quantum discord are plotted for $p=0.15$. (a) $p=0.23$ (b) against the dimensionless parameters $t \Gamma_{i} i=A, B$. In both (a) and (b), The green (against $t \Gamma_{A}$ ) and the blue (against $t \Gamma_{B}$ ) curves represent the behavior of negativity and the black (against $t \Gamma_{A}$ ) and the red (against $t \Gamma_{B}$ ) curves represent the dynamics of quantum discord.

zero in the asymptotic limit. On the other hand, the influence of qutrit's environment causes nonanalytic changes in discord. Initially it causes discord to monotonically decay reaching a minimum, abruptly increases at a critical time reaching a maximum, again start decreasing monotonically and then adopting behavior similar to the case of qubit coupling with its environment as mentioned above. Since discord is a continuous function of the plotting parameters, the abrupt increase in its dynamics at a critical point may results due to the spontaneous generated coherence between the two excited level of the qutrit system. The same argument applies to the generation of dicord in figure 2 and figure 4 as discussed next. Another notable aspect is the complete loss of quantum discord in the asymptotic limit. The amplitude damping noise transforms the initial quantum state into a classical-quantum state void of nonclassical correlations when coupled with the system for long enough time. A more or less similar behavior of quantum correlation is noted for $p=0.23$ which is shown in figure $3 b$. In figure $4(a, b)$, we plot the behaviors of quantum correlation in the presence of multilocal environments for the same two values of $p$. It can be 


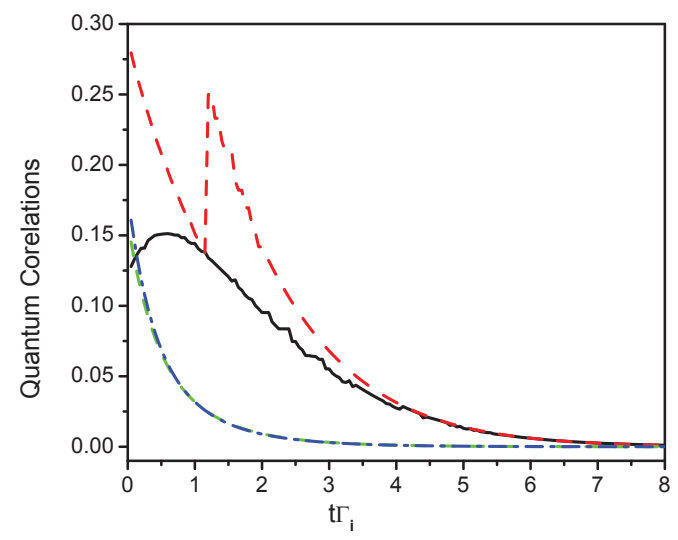

(a)

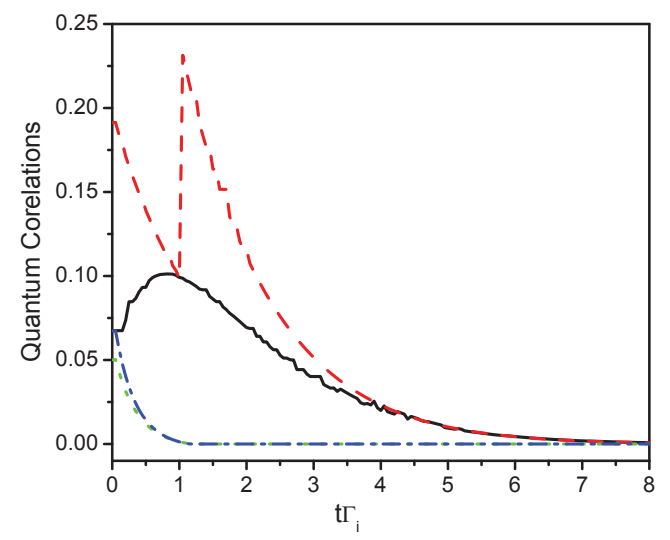

(b)

FIG. 4: (Color Online) Both the negativity and the quantum discord are plotted for $p=0.15$. (a) $p=0.23$ (b) against the dimensionless parameters $t \Gamma_{i} i=A, B$. In both (a) and (b), The green (against $t \Gamma_{A}, \Gamma_{B}=2$ ) and the blue (against $t \Gamma_{B}, \Gamma_{A}=2$ ) curves represent the behavior of negativity and the black (against $t \Gamma_{A}, \Gamma_{B}=2$ ) and the red (against $t \Gamma_{B}, \Gamma_{A}=0.2$ )curves represent the dynamics of quantum discord.

seen that overall the qualitative behavior of quantum correlations in the presence multilocal environments is unchanged, however, the peaks height of quantum discord are considerably reduced. Secondly, for both values of $p$ the quantum discord plotted against decay constant of qutrit's environment is either large or equal to the one plotted against the decay constant of qubit's environment.

\section{SUMMARY}

In this paper we report the influence of noise on the dynamics of entanglement and quantum discord in a one parameter qubit-qutrit systems. In particular, we have considered the effects of phase damping and amplitude damping noises in the Kruas operator formalism. Although the results are discussed in detail in Section IV, we summarize our findings in this section. We have analytically demonstrated that both the one way and 
two ways coupling of the environments with the system cause entanglement sudden death. The quantum discord and hence the nonclassical correlations survives when the system evolves under dephasing noise even in the asymptomatic limit. We find that quantum discord not only show robustness in coupling with qutrit's dephasing noise but can also be generated by the qutrit's dephasing noise when each marginal system is locally coupled to its own dephasing noise. On the other hand, it is found that in two ways coupling discord is very fragile against the qubit's environment. Under such coupling, the invariance and freezing behavior of quantum discord become conditional. In the case of amplitude damping noise, the behavior of entanglement is identical in both one way and two ways coupling of the marginal systems. However, the dynamics of quantum discord strongly depends on which marginal system is coupled locally to the environment. Amplitude damping noise generates discord, however, in the case of qubit coupling with environment, it is continuously generated whereas in the case of qutrit coupling the generation results in a discontinuous manner.

[1] R. Horodecki, P. Horodecki, M. Horodecki and K. Horodecki, Rev. Mod. Phys. 81865 (2009).

[2] C. H. Bennett, D. P. DiVincenzo, J. A. Smolin and W. K. Wootters, Phys. Rev. A 54, 3824 (1996).

[3] M. Horodecki, Quantum Inf. Comput. I, 3 (2001).

[4] W. K. Wootters, Quantum Inf. Comput. I, 27 (2001).

[5] R. F. Werner, Phys. Rev. A 40, 4277 (1989).

[6] H. Ollivier and W. H. Zurek, Phys. Rev. Lett. 88, 017901 (2001); W. H. Zurek, Rev. Mod. Phys. 75, 715 (2003).

[7] L. Henderson, and V. J. Vedral, Phys. A 34, 6899 (2001).

[8] A. Datta, A. Shaji, and C.M. Caves, Phys. Rev. Lett. 100, 050502 (2008).

[9] M. Gu, H. M. Chrzanowski, S. M. Assad, T. Symul, K. Modi, T. C. Ralph, V. Vedral, and P. K. Lam, Nat. Phys. 8, 671 (2012).

[10] V. Madhok and A. Datta, Phys. Rev. A 83, 032323 (2011).

[11] D. Cavalcanti, L. Aolita, S. Boixo, K. Modi, M. Piani, and A. Winter, Phys. Rev. A 83, 032324 
(2011).

[12] G. Adesso and A. Datta, Phys. Rev. Lett. 105, 030501 (2010).

[13] P. Giorda and M. G. A. Paris, Phys. Rev. Lett. 105, 020503 (2010).

[14] R. Tatham, L. Mista, Jr., G. Adesso, and N. Korolkova, Phys. Rev. A 85, 022326 (2012).

[15] J. Doukas, E. G. Brown, A. Dragan and R. B. Mann, Phys. Rev. A 87012306 (2013).

[16] S. Luo, Phys. Rev. A 77, 042303 (2008).

[17] A. Datta and S. Gharibian, Phys. Rev. A 79, 042325 (2009).

[18] A. Shabani and D. A. Lidar, Phys. Rev. Lett. 102, 100402 (2009).

[19] M. Ali, A. R. P. Rau, and G. Alber, Phys. Rev. A 81, 042105 (2010).

[20] K. Modi, T. Paterek, W. Son, V. Vedral, and M. Williamson, Phys. Rev. Lett. 104, 080501 (2010).

[21] R. Dillenschneider, Phys. Rev. B 78, 224413 (2008).

[22] M. S. Sarandy, Phys. Rev. A 80, 022108 (2009).

[23] T. Werlang and G. Rigolin, Phys. Rev. A 81, 044101 (2010).

[24] J. Maziero, H. C. Guzman, L. C. Celeri, M. S. Sarandy, and R. M. Serra, Phys. Rev. A 82, $012106(2010)$.

[25] L. Ciliberti, R. Rossignoli, and N. Canosa, Phys. Rev. A 82, 042316 (2010).

[26] B Cakmak and Z Gedik, J. Phys. A: Math. Theor. 46465302 (2013).

[27] S. R. Keshari, C. M. Caves, and T. C. Ralph, Phys. Rev. A 87, 012119 (2013).

[28] T. Yu and J. H. Eberly, Phys. Rev. Lett. 93140404 (2004).

[29] S. Khan, Math. Struct. in Comp. Science, 23, 1220 (2013).

[30] K.K. Sharma, S. K. Awasthi, and S. N. Pandey, Quantum Inf Process 123437 (2013).

[31] N. Nayak, B. Ghosh, and A. S. Majumadar, Indian Journal of Physics 84, 1039 (2010).

[32] T. Werlang, S. Souza, F. F. Fanchini and C. J. V. Boas Phys. Rev. A 80024103 (2009).

[33] L. Mazzola, J. Piilo and S. Maniscalco Phys. Rev. Lett. 104200401 (2010).

[34] Haikka P, Johnson T H and Maniscalco S 2012 arXiv:1203.6469v2.

[35] G. Karpat and Z. Gedik, Physics Letters A 3754166 (2011)

[36] G. Karpat and Z. Gedik, Phys. Scr. T153 014036 (2013). 